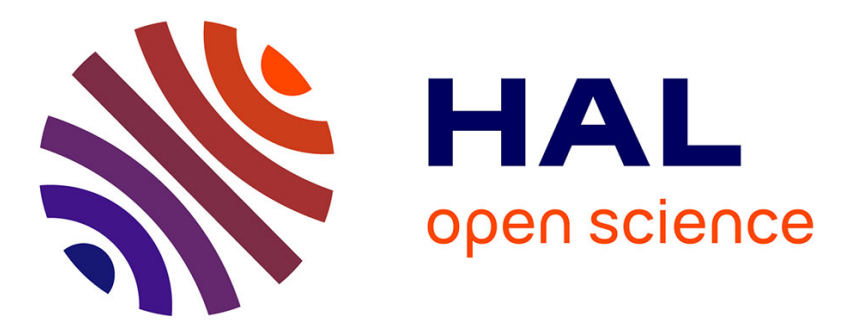

\title{
Rogue wave early warning through spectral measurements?
}

N. Akhmediev, A. Ankiewicz, J.M. Soto-Crespo, J.M. Dudley

\section{To cite this version:}

N. Akhmediev, A. Ankiewicz, J.M. Soto-Crespo, J.M. Dudley. Rogue wave early warning through spectral measurements?. Modern Physics Letters A, 2011, 375 (3), pp.541-544. 10.1016/j.physleta.2010.12.027 . hal-00582937

\section{HAL Id: hal-00582937 https://hal.science/hal-00582937}

Submitted on 21 Apr 2021

HAL is a multi-disciplinary open access archive for the deposit and dissemination of scientific research documents, whether they are published or not. The documents may come from teaching and research institutions in France or abroad, or from public or private research centers.
L'archive ouverte pluridisciplinaire HAL, est destinée au dépôt et à la diffusion de documents scientifiques de niveau recherche, publiés ou non, émanant des établissements d'enseignement et de recherche français ou étrangers, des laboratoires publics ou privés.

\section{(c)(1)}

Distributed under a Creative Commons Attribution| 4.0 International License 


\title{
Rogue wave early warning through spectral measurements?
}

\author{
Nail Akhmediev a ${ }^{\text {Adrian Ankiewicz }}{ }^{\text {, }}$ J.M. Soto-Crespo ${ }^{\text {b,* }}$, John M. Dudley ${ }^{\mathrm{c}}$ \\ a Optical Sciences Group, Research School of Physics and Engineering, Institute of Advanced Studies, The Australian National University, Canberra ACT 0200, Australia \\ ${ }^{\mathrm{b}}$ Instituto de Óptica, C.S.I.C., Serrano 121, 28006 Madrid, Spain \\ c Institut FEMTO-ST, UMR 6174 CNRS, Université de Franche-Comté, 25030 Besançon, France
}

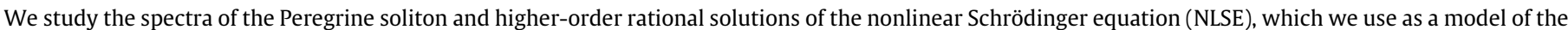

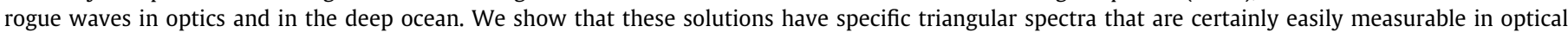

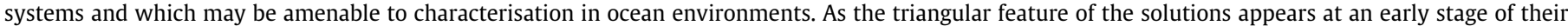

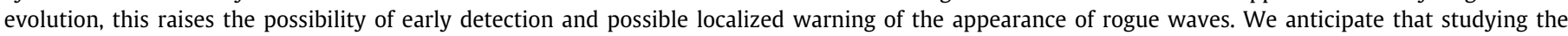
characteristics of "early warning spectra" of rogue waves may become an important future field of research.

\section{Introduction}

Rogue waves in the ocean are well known to have disastrous consequences $[1,2]$. They appear both in the deep ocean and in shallow waters [3]. In contrast to tsunamis and storms associated with typhoons that can be predicted hours (sometimes days) in advance, the particular danger of oceanic rogue waves is their sudden appearance as "waves that appear from nowhere" only seconds before they hit a ship. The grim reality, however, is that although their existence has now been confirmed by multiple different means, there remains uncertainty about their fundamental origins which hinders systematic attempts to study their characteristics and perhaps predict their appearance [4].

Clearly, large waves in the ocean can arise from multiple causes, but there is an increasing consensus that nonlinear wave shaping effects play a central role under many conditions to increase the wave amplitude. This idea has recently received much attention motivated by studies in optical fiber $[5,6]$ where the dynamics are described by the same nonlinear Schrödinger equation (NLSE) as proposed to describe the group envelope of deep water waves. As a basis, if we take the model where rogue waves are described by the NLSE, then one of the prototypes of the rogue wave is the Peregrine soliton [7-12]. Higher-order rational solutions also exist [13-15] and they can also be associated with extreme amplitudes and rogue wave characteristics [16,17]; indeed, they possess higher amplitudes than the Peregrine soliton.

\footnotetext{
* Corresponding author.

E-mail address: iodsc09@io.cfmac.csic.es (J.M. Soto-Crespo).
}

Despite the importance of these rational solutions in NLSE theory and in studies of rogue waves, they have surprisingly been the subject of limited attention. Recently, however, optical experiments have studied the excitation conditions of the Peregrine soliton in detail, confirmed its existence experimentally, and explicitly characterised its two-dimensional localisation [18]. Of course, previous experiments in wave tanks have studied related nonlinear wave propagation mechanisms [19-22], but the experiments in optics have enabled the measurement of unambiguous signatures of the defining features of the Peregrine soliton.

A particular aspect of experiments in optics is the ease of measuring optical spectra of pulse envelopes using readily-available instruments such as grating spectrometers. This has led naturally to renewed interest into the frequency-domain characteristics of the Peregrine soliton, and spectral measurements of the soliton pulse envelope have revealed a characteristic triangular form when plotted on a $\log$ scale. Significantly, theory predicts that the development of the triangular form of the envelope spectrum is an important and universal feature of parametric nonlinear spectral broadening, and indeed experiments studying the emergence of spontaneous nonlinear breather pulses from modulation instability in optics have also exhibited triangular spectra in agreement with theory [23].

These results from optics have naturally raised the question whether such spectral measurements would be possible in the ocean, and also whether such observations could provide important signatures of nonlinear effects favoring rogue wave formation. In fact, analysis of the spectral content of the full spectrum of waves on the surface of the ocean has already been studied in the context of the nonlinear wave propagation, and the BenjaminFeir Index has been introduced as a measure of how the frequency 
content of the wave spectrum relates to the frequency band of instability gain $[22,24,25]$. This can indeed be applied in a significant forecasting context to identify sea state conditions that may be associated with an elevated probability for rogue wave appearance, providing important information allowing avoidance of particular ocean regions when mapping navigation routes.

This approach, however, is based on analysis of the spectral characteristics of the sea-state as a whole. Motivated by the experiments in optics discussed above, however, we focus here on how the spectral characteristics of the envelope of propagating waves could be used to reveal the presence of nonlinearity and rogue waves a short time before the appearance of a particular rogue wave event. That is, we are interested in particular signatures of the ocean wave dynamics that can be used as "early warning" indicators in all already agitated sea. Specifically, by considering real-rime spectral analysis of the wave envelope, the appearance of characteristic triangular spectral decay of the wings could provide valuable advance and immediate warning of nonlinear amplification conditions and the emergence of rogue waves. If such spectral signatures were apparent before visual observation, then this could be extremely important in allowing appropriate evasive action to be taken.

Significantly, there is already important evidence linking such spectral decay and rogue wave formation on a smaller scale with capillary waves [26]. The open ocean, of course, presents additional difficulties, and precisely how spectral measurements could be accomplished in practice through sensing and time-series measurements of ship displacement is beyond the scope of this Letter. However, it is clearly an important question to address, and our goal here is to make the first steps in this direction. In particular, we review the properties of the characteristic triangular spectrum for rational soliton solutions of the NLSE and demonstrate that such characteristics are universal, also being observed for higherorder rogue wave solutions. By showing how these spectral characteristics appear at an early stage of evolution, our analysis supports the idea that analysing the shape of propagating waves in the frequency domain may provide important information about the emergence phase of nonlinear wave shaping mechanisms.

\section{Spectra of rogue waves}

There are several models for the description of rogue waves, both in optics and marine studies. In the lowest-order approximation, waves in the deep ocean are described by the nonlinear Schrödinger equation (NLSE). Here we start the analysis with the NLSE in dimensionless form:

$i u_{z}+\frac{1}{2} u_{t t}+|u|^{2} u=0$,

where $z$ is the longitudinal distance, $t$ is the retarded time in the frame moving with the group velocity of the waves and $|u(x, t)|$ is the wave envelope. This notation is valid for both fibre optics and ocean wave theory.

One of the NLSE solutions is the Peregrine soliton, suggested as a prototype of rogue wave as early as 1983 [7]. In our scaling, it is given by:

$u(z, t)=\left[4 \frac{1+2 i t}{1+4 t^{2}+4 z^{2}}-1\right] e^{i z}$.

It is a solution that is localised both along $z$ and $t$ directions (see upper left panel in Fig. 1). There is no periodicity and the spectrum must be a continuous function of frequency, rather than having a comb structure.

The Fourier transform of Eq. (2) can be easily calculated:

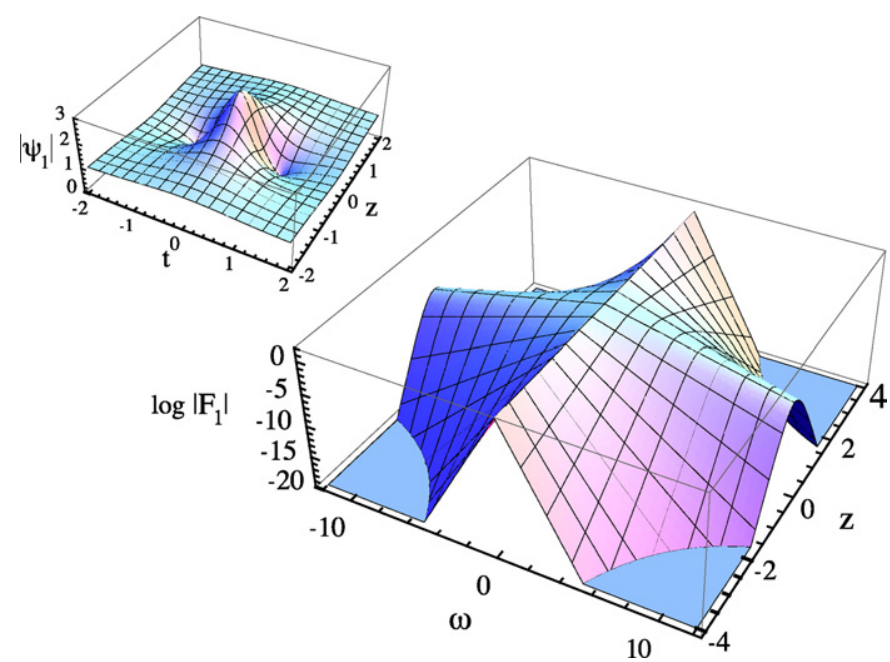

Fig. 1. (Color online.) (Upper left panel): Fundamental $(j=1)$ rogue wave (Peregrine soliton). (Bottom right panel): The spectrum of the fundamental rogue wave, plotted on a $\log$ scale i.e. $\log \left|F_{1}(\omega, z)\right|$. The always-present delta function at $\omega=0$ is omitted here.

$$
\begin{aligned}
& F(\omega, z) \\
& \quad=\frac{1}{\sqrt{2 \pi}} \int_{-\infty}^{\infty} u(z, t) e^{i \omega t} d t \\
& \quad=\sqrt{2 \pi}\left[\frac{1+2 i z}{\sqrt{1+4 z^{2}}} \exp \left(-\frac{|\omega|}{2} \sqrt{1+4 z^{2}}\right)-\delta(\omega)\right] \exp (i z),
\end{aligned}
$$

where the Dirac delta function, $\delta(\omega)$, appears due to the finite background level. The modulus of this spectrum is given by:

$$
F_{1}(\omega, z)^{\mid}=\sqrt{2 \pi} \exp \left(-\frac{|\omega|}{2} \sqrt{1+4 z^{2}}\right) .
$$

The delta function is omitted here, so Eq. (4) represents the spectrum of the variable part of the solution. This spectrum is shown in Fig. 1 on a log scale. The width of the spectrum evolves from zero at $z \rightarrow-\infty$ to a finite value at $z=0$ and decreases again at $z \rightarrow \infty$. Remarkably, at any particular $z$, the spectrum has a triangular shape and the experiments in optics referred to previously have now confirmed this prediction quantitatively [18].

\section{The spectra of higher-order rogue waves}

A second order rogue wave solution was first presented in [13] (see also [16]). This solution can be considered as a nonlinear superposition of two Peregrine solitons. There is a whole hierarchy of these solutions, with increasing order, which can be constructed using various tools. In principle, the hierarchy of higher-order polynomial solutions of the NLSE can be written in terms of Wronskians [14]. Although quite general, solutions in this form still need to be simplified, because the determinants generally contain extra factors in the form of polynomials that have to be separated and cancelled, and this is not a trivial task, even for the second order solutions. On the other hand, for any order $j$, the most convenient explicit way to write the polynomial solutions is the following [16]:

$u_{j}(z, t)=\left[\frac{G_{j}(z, t)+i z H_{j}(z, t)}{D_{j}(z, t)}+(-1)^{j}\right] e^{i z}$,

where $G_{2}(z, t), H_{2}(z, t)$ and $D_{2}(z, t)$ are polynomials in the 2 variables $z$ and $t=T / \sqrt{\left|\beta_{2}\right| L_{N L}}$ : 

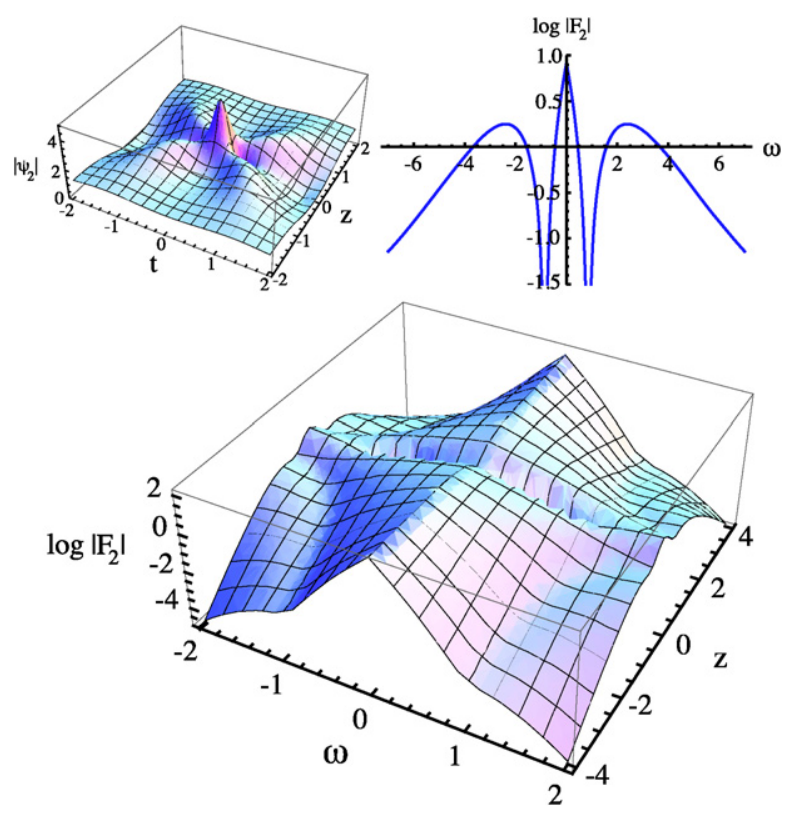

Fig. 2. (Color online.) (Above left panel): second-order $(j=2)$ rogue wave. (Bottom panel): spectrum of second order $(j=2)$ rogue wave on $\log$ scale, $\log \left|F_{2}\right|$, during evolution along the $z$-axis. (Above right panel): same spectrum on the central line, $z=0$.

$$
\begin{aligned}
G_{2}= & -\frac{3}{16}+\frac{3}{2} t^{2}+t^{4}+\frac{9}{2} z^{2}+6 t^{2} z^{2}+5 z^{4} \\
= & \left(t^{2}+z^{2}+\frac{3}{4}\right)\left(t^{2}+5 z^{2}+\frac{3}{4}\right)-\frac{3}{4}, \\
H_{2}= & -\frac{15}{8} z-3 t^{2} z+2 t^{4} z+z^{3}+4 t^{2} z^{3}+2 z^{5} \\
= & z\left(z^{2}-3 t^{2}+2\left(t^{2}+z^{2}\right)^{2}-\frac{15}{8}\right), \\
D_{2}= & \frac{3}{64}+\frac{9 t^{2}}{16}+\frac{t^{4}}{4}+\frac{t^{6}}{3}+\frac{33}{16} z^{2} \\
& -\frac{3}{2} t^{2} z^{2}+t^{4} z^{2}+\frac{9}{4} z^{4}+t^{2} z^{4}+\frac{z^{6}}{3} \\
= & \frac{1}{3}\left(t^{2}+z^{2}\right)^{3}+\frac{1}{4}\left(t^{2}-3 z^{2}\right)^{2} \\
& +\frac{3}{64}\left(12 t^{2}+44 z^{2}+1\right) .
\end{aligned}
$$

With the usual Fourier transform [FT] definition, Eq. (3), we can obtain the spectra of higher-order rogue waves, Eq. (5). Each spectrum contains a delta-function due to the constant background part of the solution. We can eliminate the delta-function by taking the Fourier transform of the varying part. Hence, we take the spectrum of

$$
\begin{aligned}
\psi(z, t) & =u_{j}(z, t) \exp [-i z]-(-1)^{j} \\
& =\left[\frac{G_{j}(z, t)+i z H_{j}(z, t)}{D_{j}(z, t)}\right] .
\end{aligned}
$$

In contrast to $u(z, t)$, the function $\psi(z, t)$ approaches zero for large $|z|$ and $|t|$.

We define $F_{j}(\omega, z)$ as the FT of the $j$-th rogue wave, i.e. the FT of the function given in Eq. (6). It is easy to find that the central value at $\omega=0$ and $z=0$ is:

$F_{j}(0,0)=\frac{1}{\sqrt{2 \pi}} \int_{-\infty}^{\infty} \frac{G_{j}(0, t)}{D_{j}(0, t)} d t=\frac{N_{j}}{\sqrt{2 \pi}}$,
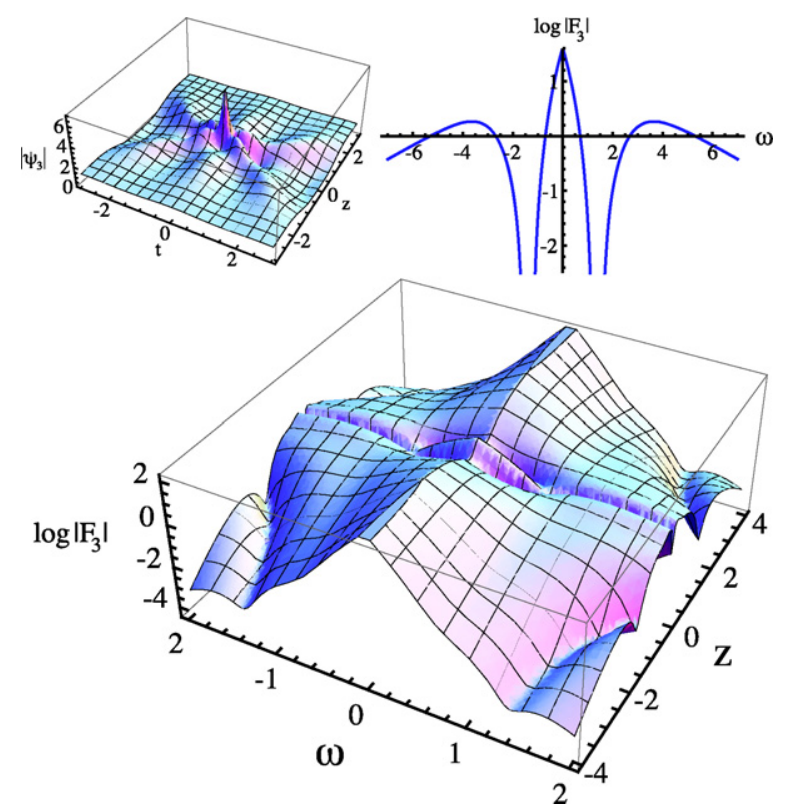

Fig. 3. (Color online.) (Above left panel): third-order $(j=3)$ rogue wave [17]. (Bottom panel): spectrum of the third order $(j=3)$ rogue wave on a $\log$ scale, $\log \left|F_{3}\right|$, showing evolution along the $z$-axis. (Above right panel): same spectrum on the central line, $z=0$.

using the definition of [17] for $N_{j}$. The above integral can be found using zeros in the upper half plane of complex $t$ [15]. Using this, we find that $\frac{F_{j}(0,0)}{\sqrt{2 \pi}}$ is equal to $(j+1) / 2$ when $j$ is odd, and equal to $-j / 2$ when $j$ is even. Thus, for the lowest-order cases, $\frac{F_{j}(0,0)}{\sqrt{2 \pi}}$ equals $(1,-1,2)$ when $j=(1,2,3)$, respectively.

For $j$ higher than one, general analytic expressions are complicated, but we can readily illustrate the results graphically. Fig. 2 shows the spectrum for the 2nd order rogue wave, along with the original profile of $|u(z, t)|$. The spectrum initially $(z=-4)$ has a shape close to being triangular. It appears to have a dip at $z=0$, but the details in the above right panel of Fig. 2 show that it has a more complicated structure at this point. Indeed, the second-order rogue wave itself has a more involved shape, and therefore so does its spectrum. On a log-scale, the spectrum has two dips, due to the fact that $F_{2}(\omega, 0)$ is zero at two values of $\omega$. Other than that the general structure of the spectrum is roughly triangular.

Explicit results for the amplitudes of higher-order rogue wave solutions have been presented in [17]. The expressions for the third-order solution are complicated and are not repeated here. Instead, we directly plot the spectrum for the 3rd order rogue wave in Fig. 3. It has a structure which is, in a way similar to, but more complicated than, the second-order result.

The results presented in this section may become useful as the study of rogue waves continues to develop. When direct measurements of wave profiles are difficult, measuring the spectral profiles can help in identifying the presence of rogue waves. As rogue waves tend to appear in various branches of physics $[3,26]$, we anticipate that developing a unified approach to their study may become extremely useful.

\section{Conclusion}

We have shown that the Peregrine soliton, which can serve as a prototype of rogue waves in the ocean has a triangular spectrum at every stage of its development. The spectra of higher order rogue waves are more complicated but can also be calculated, and we have presented spectra for the three lowest order solutions. Although more structured than the spectra for the Peregrine soli- 
ton, these higher-order solutions present characteristics that are approximately triangular when away from the zero frequency position.

A particular consequence of the results we present here relates to the insight we provide into the evolution of the spectra of these solutions. This is because, within an oceanographic context, our results suggest the potentially extremely important prospect of identifying spectral signatures of the early emergence of rogue waves resulting from nonlinear wave shaping. By linking the appearance of triangular spectra to the generation of high amplitude localised rogue wave structures, our results may provide a fruitful direction for further research aiming to identify characteristic signatures of the conditions from which rogue waves might emerge.

\section{Acknowledgements}

N.A. is grateful to Alexander von Humboldt Foundation as this work was prepared while he was visiting Germany. A.A. acknowledges the support of the Australian Research Council (Discovery Project DP0985394). J.M.S.C. acknowledges support from the Spanish Ministerio de Ciencia e Innovación under contracts FIS200603376 and FIS2009-09895. J.M.D. thanks the Institut Universitaire de France and the French Agence Nationale de la Recherche projects MANUREVA ANR-08-SYSC-019 and IMFINI ANR-09-BLAN0065, for support.

\section{References}

[1] L. Draper, Mar. Obs. 35 (1965) 193.

[2] P. Müller, Ch. Garrett, A. Osborne, Oceanography 18 (3) (2005).
[3] N. Akhmediev, E. Pelinovsky (Eds.), Rogue Waves - Towards a Unifying Concept, Special Issue of Eur. Phys. J. Special Topics 185 (2010).

[4] E. Pelinovsky, C. Kharif (Eds.), Extreme Ocean Waves, Springer, Berlin, 2008.

[5] J.M. Dudley, G. Genty, B. Eggleton, Optics Express 16 (2008) 3644.

[6] G. Genty, J.M. Dudley, B.J. Eggleton, Appl. Phys. B 94 (2009) 187.

[7] D.H. Peregrine, J. Australian Math. Soc. Ser. B 25 (1983) 16.

[8] K.B. Dysthe, K. Trulsen, Physica Scripta T 82 (1999) 48.

[9] V.V. Voronovich, V.I. Shrira, G. Thomas, J. Fluid Mech. 604 (2008) 263.

[10] I. Ten, H. Tomita, Simulation of the ocean waves and appearance of freak waves, in: Reports of RIAM Symposium No. 17SP1-2, Proceedings of a Symposium held at Chikushi Campus, Kyushu University, Kasuga, Fukuoka, Japan, March 10-11, 2006.

[11] D. Clamond, M. Francius, J. Grue, C. Kharif, European J. Mech. B - Fluids 25 (2006) 536.

[12] V.I. Shrira, V.V. Geogjaev, J. Eng. Math. 67 (2010) 11.

[13] N. Akhmediev, V.M. Eleonskii, N.E. Kulagin, Sov. Phys. JETP 62 (1985) 894.

[14] P. Dubard, P. Gaillard, C. Kleina, V.B. Matveev, Eur. Phys. J. Special Topics 185 (2010) 247.

[15] Adrian Ankiewicz, P.A. Clarkson, Nail Akhmediev, J. Phys. A: Math. Theor. 43 (2010) 122002.

[16] N. Akhmediev, A. Ankiewicz, M. Taki, Phys. Lett. A 373 (2009) 675.

[17] N. Akhmediev, A. Ankiewicz, J.M. Soto-Crespo, Phys. Rev. E 80 (2009) 026601.

[18] B. Kibler, J. Fatome, C. Finot, G. Millot, F. Dias, G. Genty, N. Akhmediev, J.M. Dudley, Nature Physics 6 (10) (2010) 790.

[19] M. Onorato, A.R. Osborne, M. Serio, L. Cavaleri, C. Brandini, C.T. Stansberg, Phys. Rev. E 70 (2004) 067302.

[20] R. Huijsmans, N. Karjanto, Andonowati, Klopman, E. van Groesen, Experiments on extreme wave generation using the soliton on finite background, in: Proceedings Rougue Waves 2004, Brest, France, 2005 Ifremer, ISBN 2-84433-150-5.

[21] N. Karjanto, E. van Groesen, J. Hydroenvironment Res. 3 (2010) 186.

[22] A.R. Osborne, Nonlinear Ocean Waves and the Inverse Scattering Transform, Elsevier, 2010.

[23] J.M. Dudley, G. Genty, F. Dias, B. Kibler, N. Akhmediev, Opt. Express 17 (2009) 21497.

[24] P.A.E.M. Janssen, J. Phys. Oceanography 33 (2003) 2001.

[25] M. Onorato, A.R. Osborne, M. Serio, Phys. Fluids 17 (2005) 078101.

[26] M. Shats, H. Punzmann, H. Xia, Phys. Rev. Lett. 104 (2010) 104503. 